\title{
ON GEODESICS OF A MODIFIED RIEMANNIAN MANIFOLD
}

\author{
D.K. Sen
}

(received May 17, 1960)

Introduction. In Riemannian geometry the autoparallels associated with the affine connexion coincide with the geodesics which arise from the metric. This is not the case in a modification of Riemannian geometry suggested by Lyra. A sufficient condition that the two classes of curves coincide is obtained.

The differential geometrical structure of a manifold is determined by

(i) an affine connexion characterized by its components $\Gamma_{\alpha \beta}^{\mu}$, which are defined by the infinitesimal parallel transfer of a vector $\xi^{\mu}$. If we let $\delta \xi^{\mu}$ denote the quantity which must be subtracted from the ordinary differential $d^{\mu} \xi^{\mu}$ in order to obtain a tensorial differential, we have

$$
\delta \xi^{\mu}=-\Gamma_{\alpha \beta}^{\mu} \xi^{\alpha} d x^{\beta} .
$$

and (ii) a metrical connexion characterized by the metric fundamental tensor $g_{\mu \lambda}$ which is defined by the measure of length 1 of a vector $\xi^{\mu}$ :

$$
1^{2}=g_{\mu \lambda} \xi^{\mu} \xi^{\lambda} \text {. }
$$

Riemannian geometry is characterized by the following as sumptions.

$$
\Gamma_{\alpha \beta}^{\mu}=\Gamma_{\beta \alpha}^{\mu} \text {, }
$$

$$
\delta 1^{2}=\delta\left(g_{\mu \lambda} \xi^{\mu} \xi^{\lambda}\right)=0 \text {, }
$$

(c)

$$
\mathrm{dg}_{\mu \lambda}=\delta_{\mathrm{g}_{\mu \lambda}} \text {. }
$$

From these it follows that

$$
\Gamma_{\alpha \beta}^{\mu}=\left\{\begin{array}{c}
\mu \\
\alpha \beta
\end{array}\right\} \text {, }
$$

where the latter quantities are Christoffel symbols of the second kind.

Canad. Math. Bull. vol. 3, no. 3, September 1960 
An autoparallel of an affine connexion is defined by a curve $x^{\mu}=x^{\mu}$ (s) (with $s$ representing arc-length), whose tangential vector $\xi^{\mu}=\mathrm{dx}^{\mu} / \mathrm{ds}$ is transferred parallel to itself. Its equation is therefore

$$
\frac{d^{2} x^{\mu}}{d s^{2}}+\Gamma_{\alpha \beta}^{\mu} \frac{d x^{\alpha}}{d s} \frac{d x^{\beta}}{d s}=0 .
$$

A geodesic of a metrical connexion, on the other hand, is defined by the extremal curves of the problem in the calculus of variations:

$$
\delta\left(\int \mathrm{ds}\right)=\delta\left(\int \sqrt{g_{\mu \lambda} \frac{d x^{\mu}}{d t} \cdot \frac{d x^{\lambda}}{d t}} d t\right)=0,
$$

where $s$ is arc-length and $t$ is an arbitrary parameter. This yields

$$
\frac{d^{2} x^{\mu}}{d s^{2}}+\left\{\begin{array}{c}
\mu \\
\alpha \beta
\end{array}\right\} \frac{d x^{\alpha}}{d s} \cdot \frac{d x^{\beta}}{d s}=0 .
$$

In view of (4), the two classes of curves are the same.

A modified Riemannian geometry. Lyra [1] suggested a modification of Riemannian geometry, which may also be considered as a modification of Weyl's geometry [3] . Weyl introduced the concept of non-integrability of length transfer, thereby modifying $(3 b)$ to

$$
\delta 1^{2}=-1^{2} \phi_{\alpha} \mathrm{dx}{ }^{\alpha}
$$

As a result

$$
\Gamma_{\alpha \beta}^{\mu}=\left\{\begin{array}{c}
\mu \\
\alpha \beta
\end{array}\right\}+\frac{1}{2}\left(\delta_{\alpha}^{\mu} \phi_{\beta}+\delta_{\beta}^{\mu} \phi_{\alpha}-g \alpha \beta \phi^{\mu}\right),
$$

where

$$
\phi^{\mu}=g^{\mu \nu} \phi_{\nu}
$$

A Weyl manifold is therefore characterized not only by $g_{\mu \lambda}$ but also by $\phi_{\alpha}$. The non-integrability of length transfer leads to the concept of gauge-transformation

$$
1^{2}+\overline{1}^{2}=\lambda\left(\mathbf{x}^{\mu}\right) 1^{2} \text {, }
$$

under which 


$$
\mathrm{g}_{\mu \lambda} \rightarrow \overline{\mathrm{g}}_{\mu \lambda}=\lambda \mathrm{g}_{\mu \lambda},
$$

(b)

$$
\phi_{\alpha} \rightarrow \bar{\phi}_{\alpha}=\phi_{\alpha}-\lambda^{-1} \frac{\partial \lambda}{\partial x^{\alpha}} \text {. }
$$

In Weyl's geometry the autoparallels and the geodesics are different.

In Lyra's geometry Weyl's concept of gauge, which is essentially a metrical concept, is modified by introducing a gauge function in the structureless manifold.

According to Lyra, the displacement vector $\overrightarrow{P P^{\prime}}$ between two neighbouring points $\mathrm{P}\left(\mathrm{x}^{\mu}\right)$ and $\mathrm{P}^{\prime}\left(\mathrm{x}^{\mu}+\mathrm{dx}^{\mu}\right)$, has the components $\xi^{\mu}=x^{0} d x^{\mu}$, where $x^{\circ}\left(x^{\mu}\right)$ is a gauge function. The coordinate system $\left(x^{\mu}\right)$ together with the gauge $x^{\circ}$ form a reference system $\left(x^{0} ; x^{\mu}\right)$. The transformation formula for a tensor under the general transformation of reference systems

$$
x^{\mu} \rightarrow x^{\mu^{\prime}}=x^{\mu^{\prime}}\left(x^{\lambda}\right) ; x^{0} \rightarrow x^{0^{\prime}}=x^{0^{\prime}}\left(x^{0}, x^{\mu}\right),
$$

with

$$
A_{\mu}^{\mu^{\prime}} \equiv \frac{\partial x^{\mu^{\prime}}}{\partial x^{\mu}} ; \quad A_{\mu^{\prime}}^{\mu} \equiv \quad \frac{\partial x^{\mu}}{\partial x^{\mu^{\prime}}},
$$

is then

$$
\xi_{\sigma_{1}^{\prime} \ldots \sigma_{r}^{\prime}}^{\rho_{1}^{\prime} \ldots \rho_{s}^{\prime}}=\lambda^{s-r} A A_{\rho_{1}}^{\rho_{1}^{\prime}} . . A_{\rho_{s}}^{\rho_{s}^{\prime}} A_{\sigma_{1}^{\prime}}^{\sigma_{1}} \ldots A_{\sigma_{r}^{\prime}}^{\sigma_{+}} \xi_{\sigma_{1} \ldots \sigma_{+}}^{\rho_{1} \ldots \rho_{s}}
$$

Thus the factor $\lambda^{s-r}$, where $\lambda=x^{\circ} / x^{\circ}$, arises as a consequence of the introduction of the gauge function.

In a Riemannian manifold the components of the affine connexion $\Gamma_{\alpha \beta}^{\mu}$ can be considered to arise as a consequence of general coordinate transformations in the following manner (cf. [4]). Let us suppose that, in a coordinate system $\left(x^{\mu}\right)$, a vector $\xi^{\mu}$ is constant, i.e. $\partial \xi^{\mu} / \partial x^{\lambda}=0$. Then, in another coordinate system $\left(x^{\mu^{\prime}}\right)$, we have

$$
\frac{\partial \xi^{\mu^{\prime}}}{\partial x^{\lambda^{\prime}}}+\Gamma_{v^{\prime} \lambda^{\prime}}^{\mu^{\prime}} \xi^{\nu^{\prime}}=0 \text {. }
$$

where

$$
\Gamma_{\nu^{\prime} \lambda^{\prime}}^{\mu^{\prime}}=-A_{\nu^{\prime}}^{\mu} A_{\mu, \lambda^{\prime}}^{\mu^{\prime}}, A_{\mu, \lambda^{\prime}}^{\mu^{\prime}} \equiv \frac{\partial}{\partial x^{\lambda^{\prime}}}\left(A_{\mu}^{\mu^{\prime}}\right) .
$$


Another way of expressing the fact that $\xi^{\mu}=$ constant would be to say that equation (11) is valid in all coordinate systems, but $\Gamma_{\nu \lambda}^{\mu}=0$ in the particular system $\left(x^{\mu}\right) . \quad \Gamma_{\nu \lambda}^{\mu}$ vanishes also in all other coordinate systems obtained by an affine transformation from this one.

We shall show that a similar analysis of a constant vector in Lyra's geometry leads to the concept of a generalized affine connexion characterized not only by $\Gamma_{\nu \lambda}^{\mu}$ but also by a function $\phi_{\alpha}$, which arises through gauge transformation.

A vector $\xi^{\mu}$ in Lyra's geometry transforms as

$$
\xi^{\mu^{\prime}}=\lambda A_{\mu}^{\mu^{\prime}} \xi^{\mu} \text {. }
$$

If $\partial \xi^{\mu} / \partial x^{\lambda}=0$ in the reference system $\left(x^{0} ; x^{\mu}\right)$, then, in the reference system $\left(\mathrm{x}^{\mathrm{O}^{\prime}} ; \mathrm{x}^{\mu^{\prime}}\right)$, we have

$$
\frac{I}{x^{O^{\prime}}} \frac{\partial \xi^{\mu^{\prime}}}{\partial x^{\lambda^{\prime}}}-\frac{I}{x^{0^{\prime}}} A_{\mu, \lambda^{\prime}}^{\mu^{\prime}} A_{\nu^{\prime}}^{\mu} \xi^{\nu^{\prime}}-\frac{I}{x^{0^{\prime}}} \frac{\partial \log \lambda}{\partial x^{\lambda^{\prime}}} \xi^{\mu^{\prime}}=0
$$

or

$$
\frac{1}{x^{0^{\prime}}} \frac{\partial \xi^{\mu^{\prime}}}{\partial x^{\lambda^{\prime}}}+\Gamma_{\nu^{\prime} \lambda^{\prime}}^{\mu^{\prime}} \xi^{\nu^{\prime}}-\frac{1}{2} \phi_{\lambda,} \xi^{\mu^{\prime}}=0
$$

whe re

$$
\Gamma_{\nu^{\prime} \lambda^{\prime}}^{\mu^{\prime}}=\frac{-1}{x^{0^{\prime}}} A_{\mu^{\prime}, \lambda^{\prime}}^{\mu^{\prime}} A_{\nu^{\prime}}^{\mu} ; \phi_{\lambda^{\prime}}=\frac{I}{x^{0^{\prime}}} \frac{\partial \log \lambda^{2}}{\partial x^{\prime}}
$$

Note that $A_{\mu}^{\mu^{\prime}} A_{\nu^{\prime}}^{\mu}=\delta_{\gamma^{\prime}}^{\mu^{\prime}}$, by $\left(10^{\prime}\right)$ and hence, by partial differentiation with respect to $x^{\prime} \lambda^{\prime}, A_{\mu, \lambda^{\prime}}^{\mu^{\prime}} A_{\nu^{\prime}}^{\mu}=-A_{\mu}^{\mu^{\prime}} A_{\nu^{\prime}, \lambda^{\prime}}^{\mu}$. Accordingly $\Gamma_{\nu^{\prime} \lambda^{\prime}}^{\mu^{\prime}}$ is symmetrical in $\nu^{\prime}$ and $\lambda^{\prime}$.

In analogy to the Riemannian case then the parallel transfer of a vector $\xi^{\mu}$ in Lyra's geometry is given by

$$
\delta \xi^{\mu}=-\left(\Gamma_{\alpha \beta}^{\mu}-\frac{1}{2} \delta_{\alpha \alpha}^{\mu} \phi_{\beta}\right) \xi^{\alpha} \mathrm{x}^{0} \mathrm{dx}{ }^{\beta} \text {. }
$$

The transformation formulae for $\Gamma_{\alpha \beta}^{\mu}$ and $\phi_{\alpha}$ are :

(i) Under coordinate transformation $x^{\mu} \rightarrow x^{\mu^{\prime}}$,

$$
\begin{aligned}
\Gamma_{\alpha \beta}^{\mu} & =A_{\mu^{\prime}}^{\mu} A_{\alpha}^{\alpha^{\prime}} A_{\beta}^{\beta^{\prime}} \Gamma_{\alpha^{\prime} \beta^{\prime}}^{\mu^{\prime}}+\frac{1}{x^{0}} A_{\gamma^{\prime}}^{\mu} A_{\alpha, \beta}^{\nu^{\prime}}, \\
\phi_{\alpha} & =A_{\alpha}^{\alpha^{\prime}} \phi_{\alpha^{\prime}} .
\end{aligned}
$$


(ii) Under gauge transformation $x^{0} \rightarrow x^{0^{\prime}}$,

$$
\begin{aligned}
\Gamma_{\alpha^{\prime} \beta^{\prime}}^{\mu^{\prime}} & =\lambda^{-I} \Gamma_{\alpha \beta}^{\mu} \quad\left(\mu^{\prime}=\mu, \alpha^{\prime}=\alpha, \beta^{\prime}=\beta\right), \\
\phi_{\alpha^{\prime}} & =\lambda^{-I}\left(\phi_{\alpha}+\frac{1}{x^{0}} \quad \frac{\partial \log \lambda^{2}}{\partial x^{\alpha}}\right) \quad\left(\alpha^{\prime}=\alpha\right) .
\end{aligned}
$$

Autoparallels of the modified manifold. An autoparallel of the generalized affine connexion is defined by a curve $x^{\tau}=x^{\tau}(s)$, whose tangential vector $\xi^{\tau}=x^{0}\left(d x^{\tau} / d s\right)$ is transferred parallel to itself. Its equation is therefore (15) $x^{0} \frac{d^{2} x^{\tau}}{d s^{2}}+\Gamma_{\lambda \mu}^{\tau} \frac{d x^{\lambda}}{d s} \frac{d x^{\mu}}{d s}\left(x^{0}\right)^{2}-\frac{1}{2}\left(\phi_{\alpha}-\dot{\phi}_{\alpha}\right) \frac{d x^{\alpha}}{d s} \frac{d x^{\tau}}{d s}\left(x^{0}\right)^{2}=0$

where

$$
\grave{\phi}_{\alpha}=\frac{1}{x^{0}} \frac{\partial \log \left(x^{0}\right)^{2}}{\partial x^{\alpha}} \text {. }
$$

A metrical connexion can be introduced in Lyra's geometry by means of a symmetric metric tensor $g_{\mu \lambda}$ :

$$
d s^{2}=g_{\mu \lambda}\left(x^{o} d x^{\mu}\right)\left(x^{o} d x\right)
$$

with the as sumption that

$$
\delta\left(g_{\mu \lambda} \xi^{\mu} \xi^{\lambda}\right)=0
$$

for arbitrary vectors $\xi^{\mu}$.

Assuming, as usual, that the process $\delta$ satisfies the product rule of differentiation and that $\delta g_{i j}=d g_{i j}$, we find, from (13) and (17), that

$$
\Gamma_{\alpha \beta}^{\mu}=\frac{1}{x^{0}}\left\{\begin{array}{c}
\mu \\
\alpha \beta
\end{array}\right\}+\frac{1}{2}\left(\delta_{\alpha}^{\mu} \phi_{\beta}+\delta_{\beta}^{\mu} \phi_{\alpha}-g_{\alpha \beta} \phi^{\mu}\right),
$$

where $\phi^{\mu} \equiv g^{\mu \lambda} \phi_{\lambda}$. A geodesic of the metrical connexion is therefore given by a solution of

$$
\delta\left(\int d s\right)=\delta \cdot\left(\int \sqrt{\left(x^{0}\right)^{2} g_{\mu \lambda} \frac{d x^{\mu}}{d t} \frac{d x}{d t}^{\lambda}} d t\right)=0,
$$

i.e. by

$$
\delta\left(\int L d t\right)=0 \text {, }
$$


where

$$
L=\sqrt{\left(x^{\circ}\right)^{2} g_{\mu \lambda} \frac{d x^{\mu}}{d t} \frac{d x^{\lambda}}{d t}} .
$$

The Euler-Lagrange equations for the problem (19) are then

$$
\frac{d}{d t}\left(\frac{\partial L}{\partial \dot{x}^{\nu}}\right)-\frac{\partial L}{\partial x^{\nu}}=0 \quad\left(\dot{x}^{\nu} \equiv \frac{d x^{\nu}}{d t}\right) \text {. }
$$

Now

$$
\frac{\partial L}{\partial x^{\nu}}=\left(\frac{d s}{d t}\right)^{-1} \frac{1}{2} \dot{x}^{\mu} \dot{x}^{\lambda}\left\{\left(x^{0}\right)^{2} g_{\mu \lambda}\right\}, \nu
$$

and

$$
\frac{\partial L}{\partial \dot{x}^{\nu}}=\left(\frac{d s}{d t}\right)^{-1}\left(x^{0}\right)^{2} g_{\mu \nu} \dot{x}^{\mu} .
$$

Substituting in the Euler-Lagrange equations, performing the differentiation and putting $t=s$, we find that the geodesics of the metrical connexion can therefore be written

$$
\left(x^{o}\right)^{2} g_{\mu \nu} \frac{d^{2} x^{\mu}}{d s^{2}}+\left(x^{o}\right)^{2}[\nu, \lambda \mu] \frac{d x^{\lambda}}{d s} \frac{d x^{\mu}}{d s}
$$

$$
+\left(2 g_{\mu \nu} x^{\circ}, \lambda-x^{\circ}, \nu g_{\mu \lambda}\right) x^{\circ} \frac{d x}{d s}{ }^{\mu} \frac{d x^{\lambda}}{d s}=0,
$$

where $[\nu, \lambda \mu]$ is the Christoffel symbol of the first kind and ${ }^{\circ}, \lambda$ denotes $\partial x^{\circ} / \partial x^{\lambda}$. Multiplying (20) by $g^{\tau \nu}$ and using (15'). we obtain

$$
\begin{aligned}
& \frac{\mathrm{d}^{2} \mathrm{x}^{\tau}}{\mathrm{d} \mathrm{s}^{2}}+\left\{\begin{array}{c}
\tau \\
\lambda_{\mu}
\end{array}\right\} \frac{\mathrm{d} \mathrm{x}^{\lambda}}{\mathrm{ds}} \frac{\mathrm{d} \mathrm{x}^{\mu}}{\mathrm{ds}} \\
& +\frac{\mathrm{x}^{\circ}}{2}\left(\delta_{\mu}^{\tau} \dot{\phi}_{\lambda}+\delta_{\lambda}^{\tau} \dot{\phi}_{\mu}-\dot{\phi}^{\tau} \mathrm{g}_{\lambda \mu}\right) \frac{\mathrm{d} \mathrm{x}^{\mu}}{\mathrm{ds}} \frac{\mathrm{dx}}{\mathrm{ds}}=0,
\end{aligned}
$$

where $\dot{\phi}^{\tau} \equiv g^{\tau \lambda} \dot{\phi}_{\lambda}$.

On the other hand, in view of (18) and (15), the equation of an autoparallel of the generalized affine connexion becomes 


$$
x^{\circ} \frac{d^{2} x^{\tau}}{d s^{2}}+\left\{\frac{1}{x^{0}}\left\{\begin{array}{c}
\tau \\
\mu \lambda
\end{array}\right\}+\frac{1}{2}\left(\delta_{\mu}^{\tau} \phi_{\lambda}+\delta_{\lambda}^{\tau} \phi_{\mu}-g_{\mu \lambda} \phi^{\tau}\right)\right\}
$$

$$
\cdot\left(x^{\circ}\right)^{2} \frac{d x^{\mu}}{d s} \frac{d x^{\lambda}}{d s}-\frac{1}{2}\left(\phi_{\alpha}-\oint_{\alpha}\right)\left(x^{0}\right)^{2} \frac{d x^{\alpha}}{d s} \frac{d x^{\tau}}{d s}=0 .
$$

A comparison of equations (21) and (22) shows that a sufficient condition that the two types of curves be the same is

$$
\phi_{\alpha}=\dot{\phi}_{\alpha} \text {. }
$$

It can easily be seen that the above condition is invariant under gauge transformations because $\dot{\phi}_{\alpha}$ transforms exactly as $\phi_{\alpha}$, when $\mathrm{x}^{\circ} \rightarrow \mathrm{x}^{\mathrm{O}^{\prime}}$.

In concluding the author wishes to acknowledge Dr. Vanstone's help with the final redaction of this paper.

\section{REFERENCES}

I. G. Lyra, Über eine Modifikation der riemannschen Geometrie, Math. Z. 54 (195I), 52-64.

2. E. Scheibe, Über einen Verallgemeinerten affinen Zusammenhang, Math. Z. 57(1952), 65-74.

3. H. Weyl, Gravitation und Elektrizität, S. -B. Preuss. Akad. Wiss, Berlin (1918), 465-480.

4. E. Schroedinger, Space-Time Structure (Cambridge, 1954), p. 27.

University of Toronto 